\title{
Cantilever surface stress sensors with single-crystalline silicon piezoresistors
}

\author{
Rasmussen, Peter Andreas; Hansen, Ole; Boisen, Anja
}

Published in:

Applied Physics Letters

Link to article, DOI:

10.1063/1.1900299

Publication date:

2005

Document Version

Publisher's PDF, also known as Version of record

Link back to DTU Orbit

Citation (APA):

Rasmussen, P. A., Hansen, O., \& Boisen, A. (2005). Cantilever surface stress sensors with single-crystalline silicon piezoresistors. Applied Physics Letters, 86(20), 203502. https://doi.org/10.1063/1.1900299

\section{General rights}

Copyright and moral rights for the publications made accessible in the public portal are retained by the authors and/or other copyright owners and it is a condition of accessing publications that users recognise and abide by the legal requirements associated with these rights.

- Users may download and print one copy of any publication from the public portal for the purpose of private study or research.

- You may not further distribute the material or use it for any profit-making activity or commercial gain

- You may freely distribute the URL identifying the publication in the public portal

If you believe that this document breaches copyright please contact us providing details, and we will remove access to the work immediately and investigate your claim 


\title{
Cantilever surface stress sensors with single-crystalline silicon piezoresistors
}

\author{
P. A. Rasmussen, ${ }^{\text {a) }}$ O. Hansen, and A. Boisen \\ Department of Micro and Nanotechnology (MIC), Technical University of Denmark, Building 345E, \\ 2800 Kgs. Lyngby, Denmark
}

(Received 8 July 2004; accepted 1 March 2005; published online 12 May 2005)

\begin{abstract}
We present a cantilever with piezoresistive readout optimized for measuring the static deflection due to isotropic surface stress on the surface of the cantilever [Sens. Actuators B 79(2-3), 115 (2001)]. To our knowledge nobody has addressed the difference in physical regimes, and its influence on cantilever sensors with integrated piezoresistive readout, that one finds between typical atomic force microscopy measurements and the surface stress sensors used in, e.g., biochemical measurements. We have simulated the response from piezoresistive cantilevers as a function of resistor type and placement for the two different regimes, i.e., surface stress measurements and force measurements. The model thus provides the means to specifically design piezoresistive cantilevers for surface stress measurements. (C) 2005 American Institute of Physics. [DOI: 10.1063/1.1900299]
\end{abstract}

Cantilevers are now widely used as chemical sensors, where the selective adsorption or immobilization of molecules on one side of the cantilever creates a surface stress difference between the two sides of the cantilever. ${ }^{1}$ This surface stress difference deforms the cantilever, and this deformation is the measured quantity. The detection principles are usually either the optical laser leverage method ${ }^{2}$ where the bending angle of the cantilever is measured or piezoresistive readout where the generated strain is measured via an integrated resistor. ${ }^{3}$

When dealing with piezoresistive readout the two key design issues determining the final resolution of the sensor are the strain sensitivity of the resistor and the electrical noise inherent in all resistors. We have previously reported on optimized cantilevers with polysilicon piezoresistors, ${ }^{4,5}$ where we focused on signal-to-noise ratio and the strain in multilayered cantilevers. In the present work we report on the optimization work on a cantilever sensor with single crystalline silicon piezoresistors made on a silicon on insulator substrate. Compared to polysilicon, the single crystalline silicon will enhance the sensitivity as its piezoresistance coefficients typically are two-three times larger and it will also improve the signal to noise ratio as the $1 / \mathrm{f}$ noise is lower. ${ }^{6}$

The optimization takes into account the different stress regimes encountered on the cantilever as a function of clamping effects, and uses the results to specify optimum design, placement, and doping of the piezoresistors. Particularly, this model clearly pinpoints the difference between cantilevers with integrated piezoresistors developed for atomic force microscopy (AFM) and biosensing, respectively.

Combined analytical and finite element (FE) work on the bending of cantilevers due to surface stress changes has been presented by Sader, ${ }^{7}$ where the effect of the clamping on the cantilever deflection is calculated for use in systems using the optical readout method. We, however, are interested in the in-plane deformation of the cantilever due to surface stress and its effect on the integrated resistor. This subject

\footnotetext{
${ }^{a)}$ Electronic mail: pra@mic.dtu.dk
}

has been the topic of recent works ${ }^{8,9}$ where a model for a piezoresistive AFM sensor is used, that is valid only for the [110] direction in $p$-type silicon on (100) wafers

$$
\frac{\Delta R}{R} \propto \pi_{L}\left(\sigma_{L}-\sigma_{T}\right)
$$

$\pi_{L}$ is the longitudinal piezoresistance coefficient and $\sigma_{L}$ and $\sigma_{T}$ are the longitudinal and transversal stress in the piezoresistor, respectively. However, this model is not suitable for describing the sensitivity to the in-plane isotropic stress.

We will use a simple approximation to analytically estimate the strain in the cantilever close to the clamping, and use a free bending model where the effect of the clamping vanishes. FE analysis will be used to check the validity of the analytical models and to calculate the precise solution to a realistic cantilever model.

When an isotropic surface stress is applied to a cantilever the unrestricted end will be subject to free bending, meaning that the strain in the cantilever is uniform and isotropic in the plane of the cantilever as long as edge effects are neglected, as sketched in Fig. 1. For comparison a cantilever with an applied force $F$ working on the apex, as found in AFM, is also pictured. In AFM the stress will be concentrated at the base of the cantilever, as the longitudinal stress $\sigma_{L}$ is given by $\sigma_{L}=z F(L-x) / I$, where $I$ is the area moment of inertia. ${ }^{10}$ The transversal stress for AFM is given by free Poisson contraction as $\sigma_{T}=-\nu \sigma_{L}$ where $\nu$ is Poisson's ratio. If we consider a resistor placed in the cantilever with the current running along the length of the cantilever, then the relative resistance change is written as the sum of a longitudinal and a transversal piezoresistive contribution

$$
\frac{\Delta R}{R}=\pi_{L} \sigma_{L}+\pi_{T} \sigma_{T}=\pi_{L} \sigma_{x}+\pi_{T} \sigma_{y}
$$

which for the free bending model with isotropic surface stress applied simplifies to 


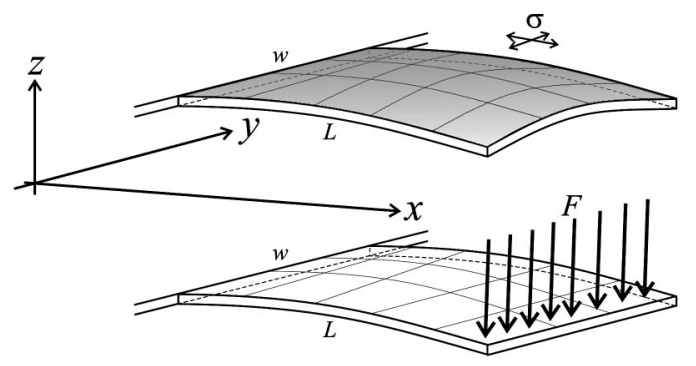

FIG. 1. Top: Cantilever with surface stress. Bottom: Cantilever experiencing a force at the apex.

$$
\frac{\Delta R}{R} \text { free }=\sigma_{L}\left(\pi_{L}+\pi_{T}\right),
$$

since $\sigma_{L}=\sigma_{T}$. It is assumed that the thickness of the cantilever is much smaller than both width and length. Thus, free Poisson contraction on practically all of the cantilever along the $z$ axis is allowed, leading to zero stress in this direction, since the effect of the clamping will only affect a region of the cantilever with a length comparable to that of the thickness. With $\sigma_{z}=0$ it then follows that $\sigma_{T}=\sigma_{y}$. Near the clamping we assume the cantilever is restricted so that there is no strain along the width of the cantilever, i.e., the strain $\varepsilon_{y}=0$, which gives $\sigma_{y}=\nu \sigma_{x} \Leftrightarrow \sigma_{T}=\nu \sigma_{L}$ leading to

$$
\frac{\Delta R}{R} \text { clamp }=\sigma_{L}\left(\pi_{L}+\nu \pi_{T}\right) \text {. }
$$

To check the validity of the analytical model it has been plotted against a FE model of a cantilever with a simulated surface stress. The exemplified cantilever is $100 \mu \mathrm{m}$ long,
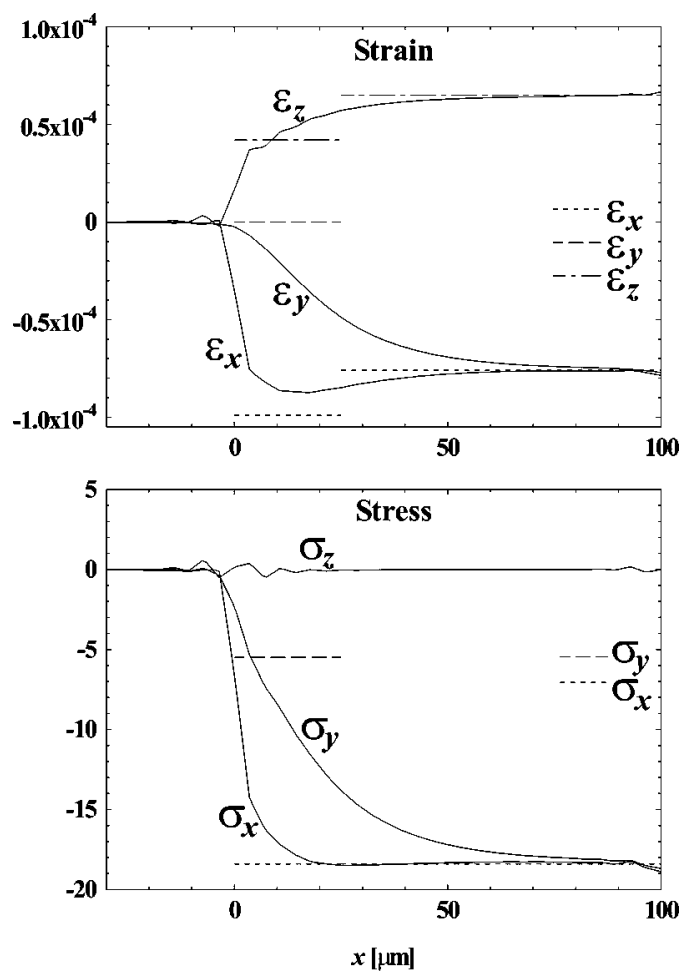

FIG. 2. Stress (MPa) and strain in the silicon just below the gold layer, in the middle of and along the length of the cantilever. $x, y$, and $z$ refer to Fig. 1 (along length, width, and thickness, respectively). The cantilever is clamped at $x=0$ and the apex is at $x=100 \mu \mathrm{m}$. The dashed lines represent the analytical solutions, with that of a clamped cantilever used for $x$

$=0-25 \mu \mathrm{m}(w / 2)$ and that of a free cantilever from $25-100 \mu \mathrm{m}$. placed from $-5 \mu \mathrm{m}$ to $x$. The integrated signals cross at $x=17 \mu \mathrm{m}$.
Downloaded 07 Jun 2010 to 192.38 .67 .112 . Redistribution subject to AlP license or copyright; see http://apl.aip.org/apl/copyright
TABLE I. Left: Longitudinal and transversal piezoresistance coefficients for the [110] direction on (100) silicon. Right: Effective piezoresistance coefficients near rigid clamping and on a free cantilever for $p$ - and $n$-type silicon.

\begin{tabular}{|c|c|c|c|c|}
\hline & & & \multicolumn{2}{|c|}{$\pi_{\text {eff }}$} \\
\hline & $\pi_{L}$ & $\pi_{T}$ & $\begin{array}{c}\text { Clamped } \\
\pi_{L}+\nu \pi_{T}\end{array}$ & $\begin{array}{c}\text { Free } \\
\pi_{L}+\pi_{T}\end{array}$ \\
\hline$p$-type & 72 & -66 & 52 & 6 \\
\hline$n$-type & -31 & $\begin{array}{c}-18 \\
{\left[10^{-11} \mathrm{~Pa}^{-1}\right]}\end{array}$ & -36 & -49 \\
\hline
\end{tabular}
A Poisson's ratio $\nu$ of 0.3 has been used.

$50 \mu \mathrm{m}$ wide, and consists of a $1 \mu \mathrm{m}$ thick silicon layer with a $0.1 \mu \mathrm{m}$ gold layer on top. In the starting position before any strain is developed in the cantilever there is a stress of $50 \mathrm{MPa}$ in the gold layer. The stress and strain distribution in the cantilever along its length axis is shown in Fig. 2. The strain $\varepsilon_{x}$ for the free cantilever is calculated analytically for a multilayered cantilever from Ref. 5. It can be seen from Fig. 2 how the analytical model fits well for distances more than the width $(50 \mu \mathrm{m})$ away from the clamping. The model obviously does not give a precise solution near the clamping, as the assumption of $\varepsilon_{y}=0$ only holds very close to the clamp. The graphs also very precisely show why optimizations using Eq. (1) give the result that the resistor should be placed as close to the clamping point as possible; when moving away from the clamp the longitudinal and transversal stresses converge and the signal becomes vanishing. Assuming we work with silicon piezoresistors on (100) silicon aligned along the $\langle 110\rangle$ directions, the longitudinal and transversal piezoresistance coefficients $\pi_{L}$ and $\pi_{T}$ can be found from $\pi_{L}=\frac{1}{2}\left(\pi_{11}+\pi_{12}+\pi_{44}\right)$ and $\pi_{T}=\frac{1}{2}\left(\pi_{11}+\pi_{12}-\pi_{44}\right)$ (Ref. 11) using Smith's ${ }^{12}$ values for the piezoresistance coefficients for low doped $p$ - and $n$-type silicon. According to Ref. 11 , the piezoresistance coefficients decrease with increasing doping concentration. However, since this trend does not differ significantly between $p$ - and $n$-type silicon, then the overall observations done with the values from Ref. 12 hold for higher doping concentrations as well. The longitudinal and transversal piezoresistance coefficients are found and listed in the left part of Table I. If these values are applied in Eqs. (3) and (4), then effective piezoresistance coefficients can be

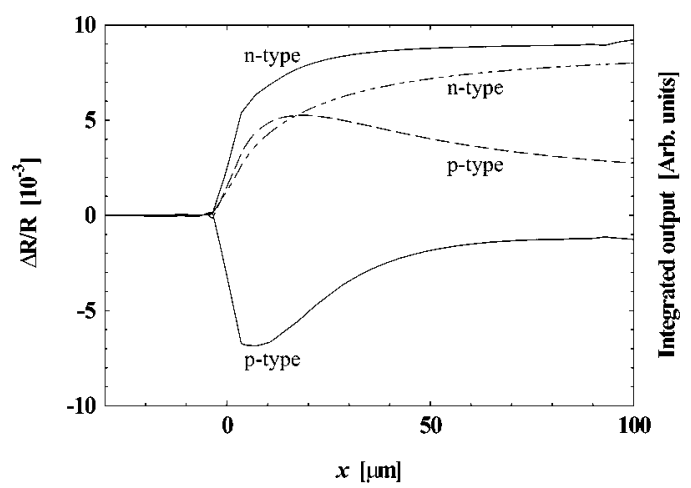

FIG. 3. $\Delta R / R$ from Eq. (2) with piezoresistance coefficients from Table I and longitudinal and transversal stress from the FE results plotted in Fig. 2. $\Delta R / R$ is plotted against the distance $x$ from the clamped end of the cantilever. The dashed lines represent the numerical value of the integrated signals, $\left(x-x_{0}\right)^{-1} \int_{x_{0}=-5 \mu \mathrm{m}}^{x}|\Delta R / R| d x$, giving the average sensitivity for a resistor

placed from $-5 \mu \mathrm{m}$ to $x$. The integrated signals cross at $x=17 \mu \mathrm{m}$.
AlP license or copyright; see http://apl.aip.org/apl/copyright.jsp 
found, and these are calculated in the two columns to the right in Table I. At the clamped edge the $p$-type silicon gives the highest sensitivity. However, as seen in Fig. 3, where FE results for the stress in the resistor from Fig. 2 are combined with the general expression from Eq. (2) to give the sensitivity $\Delta R / R$, the placement of the resistor is very critical. It is seen how the sensitivity for the $p$-type sensor is low at the free bending part of the cantilever, so the more the resistor is extended from the clamped edge of the cantilever, the less signal per length is obtained. The dashed lines show a weighed integration $\left(x-x_{0}\right)^{-1} \int_{x_{0}}^{x}|\Delta R / R| d x$ over the two sensitivity signals and for this specific example it is seen how the $n$-type resistor will give the largest signal for $17 \mu \mathrm{m}$ resistors and longer. Below $17 \mu \mathrm{m}$ the $p$-type resistor will give a signal that is marginally higher than that of the $n$-type resistor. For the free bending part of the cantilever the n-type resistor is by far the most sensitive. Since the free bending condition is more easily fulfilled than the clamped, then for any practical device the $n$-type silicon will be the first choice for a sensitive cantilever surface stress sensor. It simply requires a long, slender beam for most of it to behave as free bending, whereas an optimum placement for the $p$-type resistor in the clamped region is delicate and most likely will result in short, low volume resistors, which will increase the electrical $1 / f$ noise.

In conclusion, it has been shown how the design of piezoresistors in cantilevers for surface stress measurements in general should be different than that for AFM probes. In AFM the stress is concentrated at the base of the cantilever and the stress is primarily directed along the length of the cantilever, whereas in biosensing the stress is uniform and isotropic. For the specific case with (100) silicon with resistors placed along the $\langle 110\rangle$ directions, an $n$-type resistor will give the most sensitive surface stress sensor.

${ }^{1}$ G. Y. Chen, T. Thundat, E. A. Wachter, and R. J. Warmack, J. Appl. Phys. 77, 3618 (1995).

${ }^{2}$ G. Meyer and N. M. Amer, Appl. Phys. Lett. 53, 1045 (1988).

${ }^{3}$ M. Tortonese, H. Yamada, R. C. Barrett, and C. F. Quate, Solid-State Sensors and Actuators, 1991, Digest of Technical Papers, TRANSDUCERS '91, 1991 International Conference, pp. 448-451.

${ }^{4}$ J. Thaysen, A. Boisen, O. Hansen, and S. Bouwstra, Sens. Actuators, A 83, 47 (2000).

${ }^{5}$ P. A. Rasmussen, J. Thaysen, O. Hansen, S. C. Eriksen, and A. Boisen, Ultramicroscopy 97, 371 (2003).

${ }^{6}$ X. Yu, J. Thaysen, O. Hansen, and A. Boisen, J. Appl. Phys. 92, 6296 (2002).

${ }^{7}$ J. E. Sader, J. Appl. Phys. 89, 2911 (2001).

${ }^{8}$ S. Kassegne, M. Madou, R. Whitten, J. Zoval, E. Mather, K. Sarkar, D. Hodko, and S. Maity, Proc. SPIE 4693, 588 (2002).

${ }^{9}$ M. Yang, X. Zhang, K. Vafai, and C. S. Ozkan, J. Micromech. Microeng. 13, 864 (2003).

${ }^{10}$ Stephen D. Senturia, Microsystem Design (Kluwer Academic, Dordrecht, The Netherlands, 2001).

${ }^{11}$ Y. Kanda, IEEE Trans. Electron Devices 29, 64 (1982).

${ }^{12}$ C. S. Smith, Phys. Rev. 94 (1953). 